\title{
Mesoscale model for fission-induced recrystallization in U-7Mo alloy
}

\author{
Linyun Liang, Zhi-Gang Mei,* Yeon Soo Kim, Bei Ye, Gerard Hofman, Mihai Anitescu, \\ and Abdellatif M. Yacout
}

Argonne National Laboratory, 9700 South Cass Avenue, Lemont, IL 60439, USA

\begin{abstract}
A mesoscale model is developed by integrating the rate theory and phase-field models and is used to study the fission-induced recrystallization in U-7Mo alloy. The rate theory model is used to predict the dislocation density and the recrystallization nuclei density due to irradiation. The predicted fission rate and temperature dependences of the dislocation density are in good agreement with experimental measurements. This information is used as input for the multiphase phase-field model to investigate the fission-induced recrystallization kinetics. The simulated recrystallization volume fraction and bubble-induced swelling agree well with experimental data. The effects of the fission rate, initial grain size, and grain morphology on the recrystallization kinetics are discussed based on an analysis of recrystallization growth rate using the modified Avrami equation. We conclude that the initial microstructure of the U-Mo fuels, especially the grain size, can be used to effectively control the rate of fission-induced recrystallization and therefore swelling.
\end{abstract}

Keywords: fission-induced recrystallization; U-7Mo alloy; phase-field model

*Corresponding author.

E-mail address: zmei@anl.gov (Z. G. Mei) 


\section{Introduction}

Because of the high uranium loading density and stable irradiation behavior, UMo alloys are under investigation as candidate fuels for future high-performance research reactors to replace the currently used high-enriched uranium (HEU) fuels with lowenriched uranium (LEU) fuels [1]. However, a major concern about its qualification is the anomalous fuel swelling at high fission density. The contribution to the swelling comes mainly from the yield of fission gases. Because of the low solubility in U-Mo alloys, fission gases, such as Xe, have a strong tendency to precipitate into bubbles at locations of many radiation defects and grain boundaries. However, the thermal-induced defects diffusion is relatively low in research reactors because of the low operation temperature (usually lower than $200{ }^{\circ} \mathrm{C}$ ). The formation and growth of fission-gas bubbles are attributed mainly to fission-enhanced diffusion [2]. Because of the high burnups pursued in U-Mo fuels, fission-induced recrystallization, also called grain subdivision, further expedites the formation and growth of gas bubbles at high fission density. The acceleration of swelling in U-Mo fuels at high fission density may be closely related to the recrystallization process. Recrystallization often starts at existing grain boundaries [3, 4]. Subsequently, the front of the recrystallized grain moves into the center of the grain and eventually consumes the entire grain. Increasing the grain size and reducing the grain boundary area in U-Mo fuels by heat treatment may lead to delayed recrystallization process and reduced fuel swelling $[5,6]$.

So far, the exact mechanism of fission-induced recrystallization is not clear. Several complex processes — such as fission gas bubble formation, and grain growth — are simultaneously involved in this phenomenon [3]. Possible mechanisms related to 
recrystallization include a decrease in the potential recrystallization sites as a result of the interaction with vacancy-impurity pairs [7], buildup of stored energy in the grains because of the irradiation damage [8], stress effects due to the introduction of the high pressure of the gas bubbles [9], instability phenomena [10], and atomic cascades induced by the fission fragments [11]. A good understanding of the initiation and development of recrystallization from either theory or experiment can effectively delay the grain subdivision process and further reduce the fuel swelling.

Several simulation techniques have been used to study the recrystallization and grain growth phenomena in various materials, including the kinetic Monte Carlo method $[12,13]$, cellular automata model [14-16], rate theory model $[3,7,17,18]$, and phasefield model [19-27]. For example, Rest et al. investigated the radiation-induced recrystallization and swelling in several fuel materials, such as $\mathrm{UO}_{2}, \mathrm{U}_{3} \mathrm{Si}_{2}$ and $\mathrm{U}-10 \mathrm{Mo}$, using rate theory model $[3,7,17,18]$. However, the rate theory model cannot be used to study the effect of initial microstructure on the recrystallization and swelling because of the lack of microstructural information in such a model. As a comparison, the phase-field model can simulate the grain microstructure at mesoscale, which describes the microstructure with spatially continuous and time-dependent phase-field variables and naturally treats the material interfaces in a diffuse-interface description. In the phase-field model, the interfaces are not tracked explicitly during the microstructural evolution; therefore, no a priori assumption regarding the complex microstructure morphology is required [28]. Based on the grain growth models used by the phase-field modeling, we can categorize the current recrystallization models into three groups: (1) grain growth model based on Fan and Chen's model where phase-field variables change independently 
without any constraint [21, 22, 27, 29, 30]; (2) grain growth model based on Steinbach and Pezzolla's model where phase-field variables are subject to the constraint of their summation must be equal to one at each position [19, 20, 24, 25, 31, 32]; and (3) grain growth model based on Kobayashi, Warren, and Carter's model where only two phasefield variables with one is phase parameter and the other is crystal orientation parameter are used $[23,26]$. These models were developed to study either static or dynamic recrystallizations. To our best knowledge, however, no microstructural evolution model has been developed for fission-induced recrystallization. The irradiation condition is believed to play an important role in the recrystallization process. Moreover, the interaction between the subgrain growth and fission-induced defects such as gas bubbles makes the recrystallization process even more complex.

In this paper, we develop a mesoscale model by integrating the rate theory model and phase-field model, and we use our model to study the fission-induced recrystallization in U-7Mo alloy. The nucleation process of recrystallized grains is not directly simulated this work. Instead, we assume that the recrystallized grains are already nucleated on the grain boundaries with the critical size and concentration of recrystallized nuclei determined by the rate theory model [17]. Experimental observations indicate that fission events can produce a high density of dislocations in metallic fuels, such as U-Mo [33]. The evolution of the dislocation is assumed to be an integral step leading to recrystallization [17]. The increased dislocation density results in a stored energy in the grains, which provides a driving force for the growth of subgrains. The grain growth behavior is then simulated by a multiphase phase-field model. During recrystallization, 
fission-gas bubbles are introduced in the grain structure, in which they either interact with the grain boundary or act as nucleation sites for recrystallization.

The remaining manuscript is organized as follows. Section 2 presents the mesoscale model for the fission-induced recrystallization including the rate theory and the phase-field models. Section 3 summarizes the material properties used by the rate theory model, which are obtained from either experimental measurements or density functional theory (DFT) calculations. Section 4 presents the computational details of the phase-field simulations. Detailed simulation results and discussion are given in Section 5. Section 6 provides conclusions and briefly discusses future work.

\section{Mesoscale model of recrystallization}

This section describes the development of a mesoscale model by integrating the rate theory model predicting the density and critical radius of the recrystallized nuclei and the dislocation density and the phase-field modeling of the subgrain growth in U-7Mo alloy.

2.1 Rate theory model of dislocation density and nucleation of recrystallization

Generally the dislocation density varies in different grains that depends on the grain size. For the UMo fuel the grain size is around several micrometers. The difference of dislocation densities in different grains is relative small due to their large grain sizes. Therefore, the dislocation density can be considered to be identical for different grains. According to Rest [17], the evolution of cellular dislocation structure is the integral step 
leading to fission-induced recrystallization. The walls of cellular structure and/or the grain boundaries of the subgrain structure are considered to be potential recrystallization nuclei. The initial dislocation density at the steady state is calculated by [17]

$$
\rho_{d}=\left(\frac{3}{L a}\right)^{2 / 3}\left(\frac{\alpha_{i}}{B_{i} D_{i}}\right)^{1 / 3}\left(\frac{K D_{v}}{\alpha_{r}}\right)^{1 / 6},
$$

where $\alpha_{i}=\sqrt{2} / a^{2}$ is a proportional constant for nucleation of loops as di-interstitials, $a$ is the lattice constant, $\alpha_{r}=4 \pi r_{i v} / \Omega$ is the rate constant for loss of defects due to recombination, $r_{i v}$ is the radius of the recombination volume, $\Omega$ is the atomic volume, $L$ is the distance between dislocation loop planes, $B_{i}$ is the relative bias between interstitials and vacancies for dislocations, $K$ is the displacement rate, and $D_{v}$ and $D_{i}$ are diffusivities of vacancy and self-interstitial atoms.

The dislocations introduced by fission can produce a long-range stress field. Such a stress field can be considered as stored energy in the grain structure, which causes structural instability and results in smaller recrystallization nuclei. The growth of subgrains is proportional to the dislocation density. The existence of precipitates can affect the sub-grain growth due to the pinning effect. The recrystallization originates by the growth of subgrains within the deformation zone on a precipitate. After the deformation zone has been consumed, a nucleus experiences a barrier to further growth. The driving force for the recrystallization arises from the dislocation density that is considerably lower in the matrix outside the deformation zone, and the recrystallization cannot proceed unless the driving force can overcome the retarding force due to the grain boundary. For a given dislocation density, there exists a critical nucleus size that below which the nucleus will not grow beyond the deformation zone. The critical size of the 
recrystallized nuclei can be estimated by the critical precipitate size based on the Particle Simulated Nucleation of Recrystallization (PSN) model [34, 35], which assumes that the radius of nucleus is taken to be half of the precipitate diameter. Base on Rest's model, the critical precipitate size providing a nucleus able to grow beyond the deformation zone can be estimated by [17]

$$
r_{c r i t}=\frac{3 \alpha_{p} f(v) \rho_{d}}{4 n_{p} \gamma \pi\left(C_{A} C_{\rho}\right)^{2}}
$$

where $\alpha_{p}$ is a constant of the order unity, $n_{p}=\phi F_{d}$ is the number of precipitate (gas bubble in this work) per unit volume, $\phi$ is the number of precipitate formed per fission event, $F_{d}$ is the fission density, $f(v)=(1-v / 2) /(1-v), v$ is Poisson's ratio, $C_{A}$ and $C_{\rho}$ are constants, and $\gamma$ is a factor that takes into account the lower force that acts on the dislocations when the precipitates are passed by climb.

Based on the rate theory model, the potential recrystallization nuclei are taken to be the nodes, or triple points of the cellular dislocation or subgrain structure. The density of the nuclei is related to the cell size, which is further related to the dislocation density by minimizing the total energy (dislocation line energy plus the stored energy). Thus, the initial concentration of recrystallization nuclei related to the dislocation density from the rate theory becomes [17]

$$
C_{r x}=\frac{\left|\rho_{d} f(v) / \pi\right|^{3 / 2}}{\left(C_{A} C_{\rho}\right)^{7}}
$$

Thus, the initial concentration and critical size of the recrystallization nuclei depend on the dislocation density, which is related to the fission rate. This predefined 
information will be used by the phase-field model in the next section for modeling the grain subdivision process.

\subsection{Phase-field modeling of recrystallization}

Once the recrystallization nuclei are nucleated, the growth kinetics is controlled by the grain growth law. In the phase-field modeling of the fission-induced recrystallization in $\mathrm{U}-7 \mathrm{Mo}$ alloy, the grain parameter $\eta_{i}(r, t)$ is chosen to describe the polycrystalline structure. $\eta_{i}(r, t)$ is equal to 1.0 inside the $i$ grains and 0 in the $j \neq i$ grains. $\eta_{i}(r, t)$ continuously changes from 1.0 to 0 across the $i$ and $j$ grain boundaries. $c_{g}$ is chosen to describe the fission gas Xe concentration, which is also used to distinguish the gas bubble phase and matrix phase. It has a value of 1.0 inside the gas bubble and 0 in the matrix, and continuously changes from 0 to 1.0 along the interface between the matrix and gas bubbles. For simplicity, the formation and diffusion of vacancy and selfinterstitial atom are not explicitly modeled in this work. The total free energy of the system can be described by

$$
F\left(c_{g}, \eta_{i}\right)=\int\left[f_{\text {bulk }}\left(c_{g}, \eta_{i}\right)+\sum_{i=1}^{i=p} \frac{\kappa_{\eta}}{2}\left|\nabla \eta_{i}\right|^{2}+\frac{\kappa_{c}}{2}\left|\nabla c_{g}\right|^{2}+f_{\text {stored }}\left(\eta_{i}\right)\right] d V
$$

where $f_{\text {bulk }}$ is the bulk free energy density describing the composition and volume fraction of the equilibrium phases; $\kappa_{\eta}$ and $\kappa_{c}$ are the gradient energy coefficients for the phase parameter and composition, respectively; $p$ represents the total number of grain orientations in a grain structure; and $f_{\text {stored }}$ is the increased dislocation density resulting in a stored energy. 
The bulk free energy used for the multiphase grain growth model is described by

$$
\begin{aligned}
f_{\text {bulk }}\left(c_{g}, \eta_{1}, \eta_{2}, \ldots, \eta_{p}\right)= & W c_{g}^{2}\left(1-c_{g}\right)^{2}+\sum_{i=1}^{p}\left(-\frac{A}{2} \eta_{i}^{2}+\frac{B}{4} \eta_{i}^{4}\right)+C \sum_{i=1}^{p} \sum_{j \neq i}^{p} \eta_{i}^{2} \eta_{j}^{2}+ \\
& \frac{1}{4}+S_{I} c_{g}\left(1-\sum_{i=1}^{p} \eta_{i}^{2}\right),
\end{aligned}
$$

where the last term describes the interactions between fission gas and grain boundaries. This term ensures that the grain parameters $\eta_{i}$ have the zero values when the gas concentration has a value of 1.0, i.e, there is no grains inside the gas bubble. Parameters $A, B, C, W$, and $S_{\mathrm{I}}$ are positive constants.

The stored elastic energy in each grain due to the dislocation is described as

$$
f_{\text {stored }}\left(\eta_{i}\right)=\frac{1}{2} \eta_{i}^{2} G b^{2} \rho_{i}
$$

where $G$ is the shear modulus, $b$ is the Burgers vector, and $\rho_{i}$ is the dislocation density for each grain.

The spatial and temporal evolutions of grain parameters and Xe concentration follow the Allen-Cahn and Cahn-Hilliard equation [36],

$$
\begin{aligned}
& \frac{\partial \eta_{i}}{\partial t}=-L_{\eta} \frac{\delta F}{\delta \eta_{i}}, i=1,2, \ldots, p . \\
& \frac{\partial c_{g}}{\partial t}=\nabla M \nabla \frac{\delta F}{\delta c_{g}}+\dot{g} .
\end{aligned}
$$

where $L_{\eta}$ is the kinetic coefficient of grain boundary movement, $M$ is the gas atom mobility, and $\dot{g}$ is the fission gas generation rate. The influence of the thermal noise is neglected in the simulation since the nucleation process is not model. 


\section{Materials properties}

Table 1 lists the parameters of the U-7Mo alloy used for calculating the dislocation density by the rate theory model and for simulating the recrystallization by the phase-field model $[3,17,18]$.

Table 1. Material properties of U-7Mo alloy used in the rate theory and phase-field models

\begin{tabular}{llll}
\hline Quantity & Symbol & Value & Reference \\
\hline Distance between planes & $L$ & $3.0 \times 10^{-8} \mathrm{~m}$ & {$[17]$} \\
Lattice constant & $a$ & $3.42 \times 10^{-10} \mathrm{~m}$ & {$[18]$} \\
Displacement rate & $K$ & $\dot{f} /\left(5.0 \times 10^{23}\right)$ & This work \\
Radius of recombination volume & $r_{\mathrm{iv}}$ & $8.25 \times 10^{-9} \mathrm{~m}$ & {$[18]$} \\
Relative bias between interstitials and vacancies & $B_{\mathrm{i}}$ & $5.0 \times 10^{-3}$ & {$[18]$} \\
Interstitial diffusivity & $D_{i}{ }^{0}$ & $3.81 \times 10^{-9} \mathrm{~m}^{2} \mathrm{~s}^{-1}$ & {$[18,37]$} \\
Vacancy diffusivity & $D_{v}{ }^{0}$ & $5.72 \times 10^{-8} \mathrm{~m}^{2} \mathrm{~s}^{-1}$ & {$[18,37]$} \\
Poisson ratio & $v$ & 0.31 & {$[17]$} \\
Constant & $C_{\mathrm{A}}$ & $3(\mathrm{for} \mathrm{cubic} \mathrm{cell)}$ & {$[17]$} \\
Constant & $C_{\rho}$ & 1.0 & {$[17]$} \\
Atomic volume & $\Omega$ & $a^{3} / 2$ & {$[17]$} \\
Interstitial migration enthalpy & $\varepsilon_{\mathrm{i}}$ & $0.27 \mathrm{eV}^{-1.0}$ & {$[18]$} \\
Vacancy migration enthalpy & $\varepsilon_{\mathrm{v}}$ & $1.08 \mathrm{eV}$ & {$[18]$} \\
Surface energy of gas bubble & $\gamma$ & $1.64 \mathrm{~J} / \mathrm{m}^{2}$ & {$[38]$} \\
Grain boundary energy & $\sigma_{g b}$ & $0.36 \mathrm{~J} / \mathrm{m}^{2}$ & {$[6]$} \\
Kinetic coefficient & $L_{\eta}$ & $1.82 \times 10^{-14} \mathrm{~m}^{3} \mathrm{~s}^{-1} \mathrm{~J}^{-1}$ & - \\
Gas atom mobility & $M$ & $4.55 \times 10^{-31} \mathrm{~m}^{5} \mathrm{~s}^{-1} \mathrm{~J}^{-1}$ & - \\
Constant & $S_{I}$ & 1.0 & - \\
Shear modulus & $G$ & $36.0 \mathrm{GP}$ & {$[6]$} \\
& & &
\end{tabular}


Burgers vector

Gradient coefficient

Constant

$$
\begin{array}{ll}
b & 3.42 \times 10^{-10} \mathrm{~m} \\
\kappa_{c} & 2.74 \times 10^{-7} \mathrm{Jm} \\
W & 1.76 \times 10^{8} \mathrm{Jm}^{-3}
\end{array}
$$

The displacement rate $K$ in $\mathrm{U}-7 \mathrm{Mo}$ is predicted to be $7.55 \mathrm{dpa} / \mathrm{s}$ by the Monte Carlo simulation code SRIM (Stopping and Range of Ions in Matter) [39, 40]. Therefore, the conversion constant $B$ in equation $K=\dot{f} / B$ is estimated to be about $5.0 \times 10^{23}$ fissions $/ \mathrm{dpa} / \mathrm{m}^{3}$.

In real polycrystalline U-7Mo fuel samples, the grain boundary energy $\sigma_{g b}$ and mobility $M$ vary from grain boundary to grain boundary, depending on the grain boundary misorientation and inclination. However, the mathematical models usually ignore the variation of grain boundary energy and mobility. A single value for $\sigma_{g b}$ and $M$ was used for all grain boundaries in the present simulations. This simplified scenario, called idea grain growth, is used extensively by computer simulation [41-44].

The input parameters for the phase-field simulations are determined as follows. According to Moelans et al. [45], the interfacial thickness $\left(l_{g b}\right)$, expansion coefficients of chemical free energy, interfacial energy $\left(\sigma_{g b}\right)$, and gradient coefficient are interrelated in the phase-field model. For a symmetrical phase parameter profile with the saddle point of the free energy located at $\eta_{i}=0.5$, the relationships can be expressed as

$$
\sigma_{g b}=\frac{\sqrt{2}}{3} \sqrt{A \kappa_{\eta}} \quad \text { and } \quad l_{g b}=\sqrt{\frac{8 \kappa_{\eta}}{A}}
$$




\section{Phase-field simulation details}

To study the statistically averaged kinetics and topological features of the ideal two-dimensional recrystallization, we performed phase-field simulations on a simple square domain. The phase-field model was implemented in an in-house simulation code, and the semi-implicit FFTW numerical method was employed to solve the coupled equations (7-8) [46]. Periodic boundary conditions were imposed on the simulation domain.

The initial gain structure was generated by using the conventional phase-field grain growth model. The initial grain size can be controlled by the evolution time. In order to simulate the recrystallization, we randomly seed the newly formed subgrains on the grain boundaries instead of modeling the nucleation process of recrystallization directly. The formed nuclei were assumed to have a circular shape. The corresponding new phase parameter was created for each recrystallized grain. The critical size and density of the nuclei at different irradiation rates and temperatures can be determined according to Eqs. (2-3). The dislocation density calculated from the rate theory model (see Eq. 1) is assumed homogeneous through the grain microstructure, which was set as zero for the newly formed sub-grains. 
Similar to the subgrain growth, the nucleation of gas bubbles was not directly modeled in this simulation. We focused on the gas bubble evolution after nucleation in order to simplify the modeling. The initial gas atom concentration was set as zero before fission events start. The nucleated gas bubbles due to the fission generated gas atoms were randomly introduced on the grain boundaries with the bubble size from $0.1 \mu \mathrm{m}$ to $0.3 \mu \mathrm{m}$. The rate of introduced gas bubbles was controlled so that its density best reproduces the fuel swelling measured by experiments [4]. The intragranular gas bubbles are neglected in this work due to the facts that they have very small sizes (usually in several nano meters) and less contribution to the overall swelling.

A model size of $25.6 \mu \mathrm{m} \times 25.6 \mu \mathrm{m}$ and the grid size $\Delta \mathrm{x}=\Delta \mathrm{y}=0.05 \mu \mathrm{m}$ were used in the simulations. The average initial grain size was around 3-10 $\mu \mathrm{m}$, which is consistent with the initial grain size measured in the U-Mo dispersion fuels [4]. The temporal evolution of grain structures with different initial grain size were studied in detail. In order to differentiate the different grains in the simulations, a function $\xi(r)=\sum_{i=1}^{p} \eta_{i}^{2}(r)$ is used, which takes a value of unity within the individual grain and a smaller value along the grain boundary region.

\section{Results and discussion}

In this section, we first estimate the initial dislocation density, critical size and density of recrystallization nuclei using the mechanistic rate theory models by Rest [17]. With this information, we conducted the phase-field simulations of radiation-induced grain subdivision processes and gas bubble evolutions in the U-7Mo alloy. 


\subsection{Dislocation density by rate theory model}

By adopting the rate theory model, the initial dislocation densities for the onset of recrystallization can be estimated using Eq. (1). The recrystallization starts when the fission density is above $2.40 \times 10^{21} \mathrm{~cm}^{-3}$ as indicated from experiments [4]. Figure 1 shows the calculated dislocation density in the irradiated U-7Mo alloy as a function of the fission rate and temperature. The predicted dislocation density is on the order of $10^{15} \mathrm{~m}^{-2}$, which is consistent with the dislocation density in the in-pile-irradiated U-7Mo fuel measured by Miao et al. [47]. We note that the experimental data was measured at slightly different temperatures and fission rates. Overall, the dislocation density in the U7Mo alloy increases with the increasing fission rate and temperature. Thus, a high fission rate and temperature may expedite the recrystallization process.
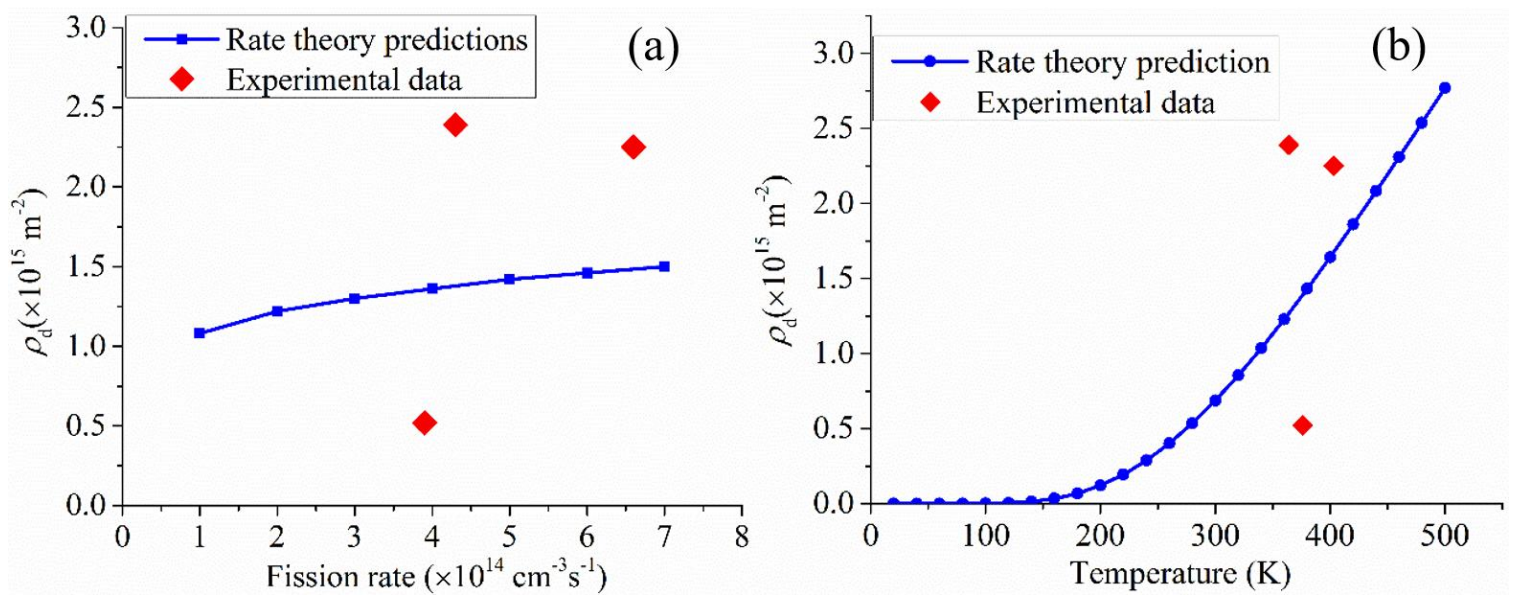

Fig. 1. Dependencies of dislocation density on fission rate at temperature of $373 \mathrm{~K}$ (a) and on temperature at fission rate of $3.0 \times 10^{14} \mathrm{~cm}^{-3} \mathrm{~s}^{-1}$ (b) predicted by the rate theory models [17]. The experimental data were measured by Miao et al. [48]. 
The critical size and density of the recrystallization nuclei were also computed by using Eqs. (2) and (3). The critical nucleation size is estimated to be $7.07 \mathrm{~nm}$, and the critical sub-grain density is predicted to be $5.64 \times 10^{13} \mathrm{~m}^{-2}$. Since the predicted critical size is much smaller than the grid size used in the current phase-field simulations, a larger critical nucleation size of $0.2 \mu \mathrm{m}$, four times larger than the grid size, was adopted in order to make the simulation feasible. In order to maintain the same total area of the nuclei, the calculated density of nuclei was set as $1.06 \times 10^{12} \mathrm{~m}^{-3}$.

\subsection{Recrystallization kinetics by the phase-field model}

With the predicted dislocation density, critical nucleation size, and density of recrystallized nuclei, the recrystallization kinetics of U-7Mo alloy were simulated by using the multiphase phase-field model. The simulated microstructure evolution in U7Mo with respect to fission density is shown in Fig. 2. The total number of initial grains in the simulation is 89 , and the average grain size in the initial microstructure is about 3.0 $\mu \mathrm{m}$. 


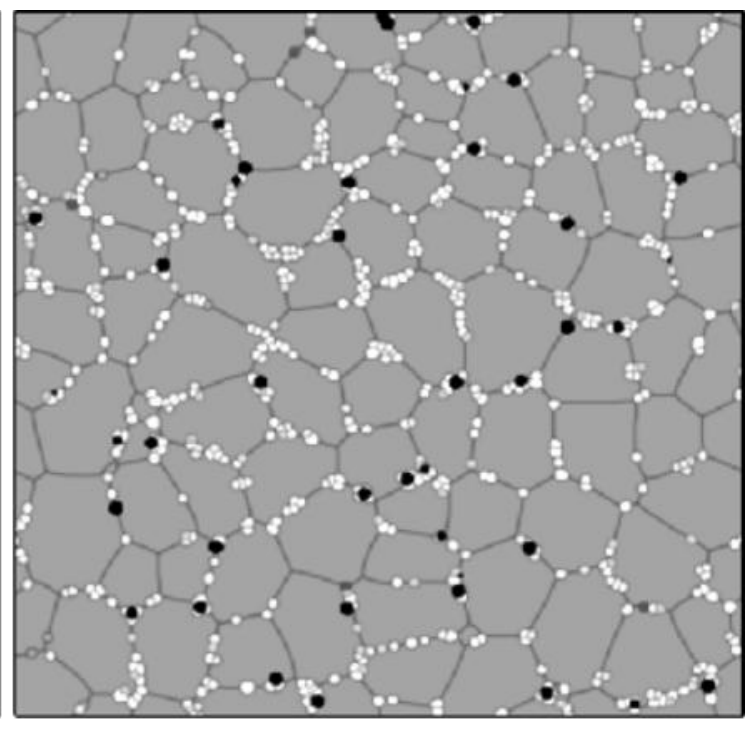

(a) $F=2.40 \times 10^{21} \mathrm{~cm}^{-3}$

(b) $F=3.10 \times 10^{21} \mathrm{~cm}^{-3}$
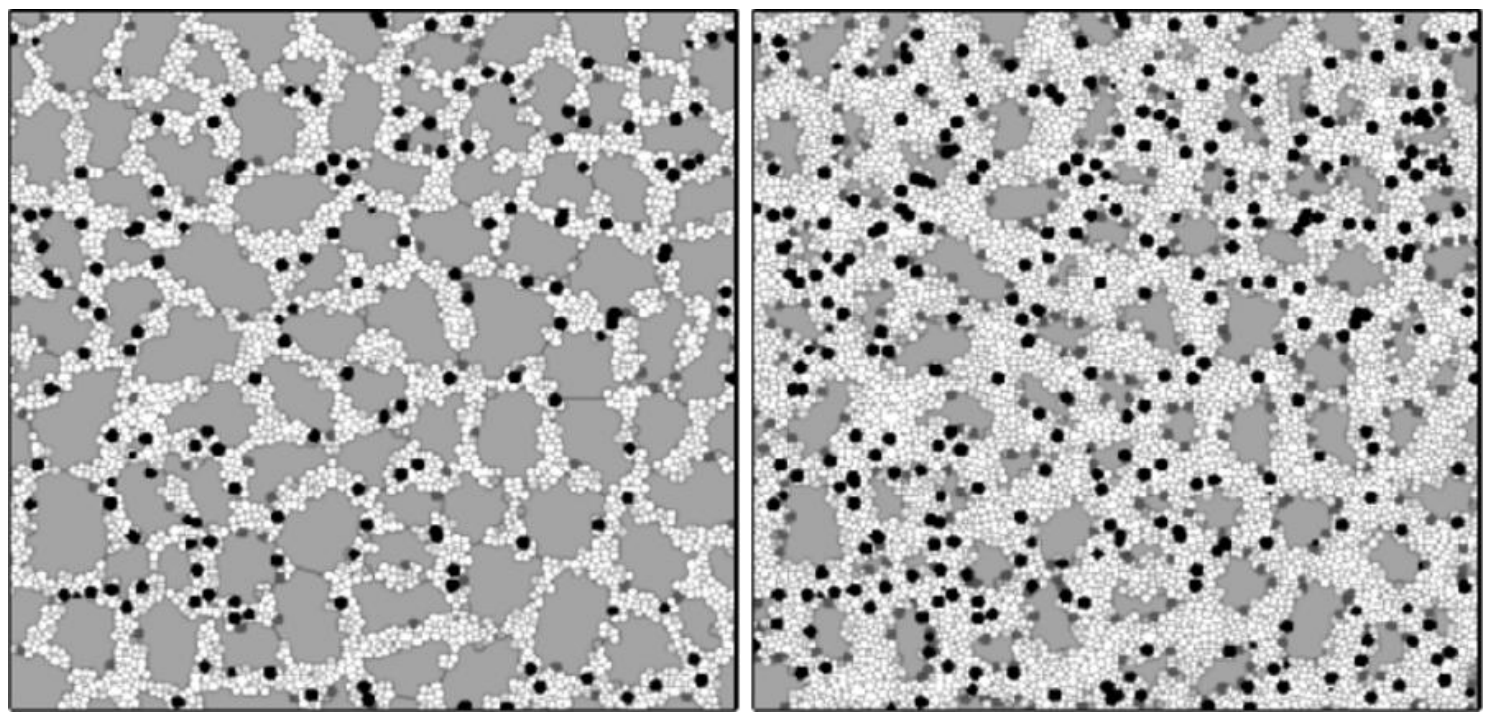

(c) $F=4.10 \times 10^{21} \mathrm{~cm}^{-3}$

(d) $F=4.80 \times 10^{21} \mathrm{~cm}^{-3}$

Fig. 2. Temporal evolution of the grain structure in U-7Mo alloys at different fission densities with the fission rate of $3.0 \times 10^{14} \mathrm{~cm}^{-3} \mathrm{~s}^{-1}$. Grey: original grains; White: subgains; Black: gas bubbles. 
The recrystallization starts from the pre-existing grain boundaries. These recrystallized grains then grow toward the center of the original grains as a result of fission. The number and size of the initial grains decrease with increased fission density. The newly formed grain boundaries become new nucleation sites for recrystallization. With increased area of grain boundaries, the number of recrystallized grains significantly increases at high fission density. The full recrystallization of the U-7Mo alloy at a fission rate of $3.0 \times 10^{14} \mathrm{~cm}^{-3} \mathrm{~s}^{-1}$ can be achieved at a high fission density around $5.5 \times 10^{21} \mathrm{~cm}^{-3}$. The grain size of fully recrystallized U-7Mo alloy ranges from $0.2 \mu \mathrm{m}$ to $0.5 \mu \mathrm{m}$ [49]. During the recrystallization process, the grain boundary energy and stored energy are the main driving forces for grain growth. The grain boundary energy drives the large grains to grow at the expense of small grains with the reduction of grain boundary energy. The stored energy in the deformed grains induces the growth of recrystallized grains. Within the recrystallized area or after the fully recrystallization, the grain growth is driven only by the grain boundary energy.

The nucleation and growth of gas bubbles are responsible for the fuel swelling. Similar to recrystallization, they prefer to nucleate on the existing grain boundaries. If two bubbles get close to each other, they can coalescence into a bigger bubble. The density of gas bubbles increases with the increasing fission density because of the increased grain boundary area. The increasing number and size of fission gas bubbles at high fission density lead to the further increase of fuel swelling.

To study the recrystallization kinetics, we calculated the recrystallized volume fraction as a function of fission density with the fission rate $\dot{f}=3.0 \times 10^{14} \mathrm{~cm}^{-3} \mathrm{~s}^{-1}$. The recrystallization volume fraction was obtained by dividing the total volume of the 
recrystallized grains and newly formed gas bubbles by the initial sample volume, an approach similar to the one used by Kim et al. [4]. To validate our model for the gas bubbles, we calculated the gas bubble-induced fuel swelling using the formula $\Delta V / V_{0}$, where $\Delta V$ is the increased volume due to the introduced gas bubbles, and $V_{0}$ is the original volume of the fuel, which is compared with the experimental measurements [4] as shown in Fig. 3(a). Good agreement between simulation and experimental results implies that the current model for gas bubble evolution is reasonable. We note that intragranular gas bubbles are not considered in this model. Therefore the total volume of gas bubbles is underestimated at low fission density since fission gases exist mainly in intragranular bubbles at low fission density. The predicted volume fraction of recrystallized grains is depicted in Fig. 3(b) together with experimental data compiled by Kim et al. [4]. Good agreement with experimental results is obtained in the whole range of fission density.
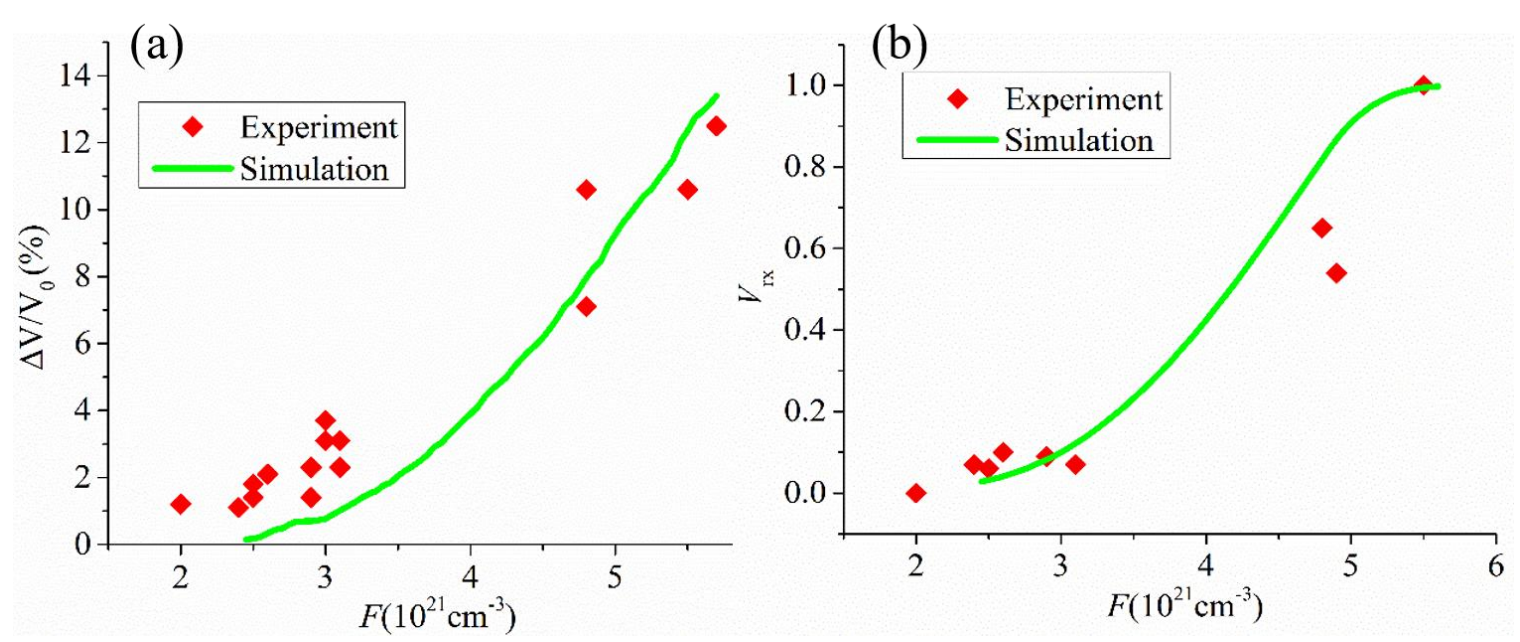
Fig. 3. Calculated fission gas bubble swelling (a) and recrystallized volume fraction, (b) in the U-7Mo alloy together with experimental data compiled by Kim et al. [4]. The fission rate is set as $3.0 \times 10^{14} \mathrm{~cm}^{-3} \mathrm{~s}^{-1}$ in the simulation. The filled diamonds represent the experimental data and the line denotes the simulation results.

Due to the simplicity of the analytical description, the Avrami model is often used to compare with the experimental measurements of recrystallization kinetics. As shown by Kim et al. [4], the irradiation-induced recrystallization process in U-Mo alloys can be described by the modified Avrami equation, also called Johnson-Mehl-AvramiKolmogorov (JMAK) equation,

$$
V_{r x}=1-\exp \left[-K\left(F-F_{0}\right)^{n}\right]
$$

where $V_{r x}$ is the fuel volume fraction with subdivided grains, $K$ is the recrystallization reaction constant related to the Mo content, $F_{0}$ is the incubation fission density below which recrystallization does not occur, and $n$ is the Avrami exponent that is dependent on the nucleation and growth mechanism of recrystallization. Experimental measurements of recrystallization kinetics are usually compared with the JMAK model by plotting $\log \left[-\ln \left(1-V_{r x}\right)\right]$ against $\log [t]$, or $\log \left[\left(F-F_{0}\right)\right]$ for fission-induced recrystallization. According to Eq. (10), this should yield a straight line of slope equal to the Avrami exponent $n$. As shown in Fig. 4(a), the calculated $\log \left[-\ln \left(1-V_{r x}\right)\right]$ shows a good linear relationship with $\log \left[\left(F-F_{0}\right)\right]$ when the fission density is lower than $4.5 \times 10^{21} \mathrm{~cm}^{-3}$. By fixing the critical fission density $F_{0}$ at $1.67 \times 10^{21} \mathrm{~cm}^{-3}$, the Avrami exponent $n$ is determined to be 2.81 , which is consistent with the value of 2.6 obtained by Kim et al. 
from the fitting of experimental data [4]. We note that the key assumption of the Avrami model is that the nucleation sites are randomly distributed. However, in real materials the nuclei sites are not randomly distributed but more preferably localized on grain boundaries. Therefore, experimental results rarely show perfect agreement with the Avrami model. The Avrami exponent is often found to decrease at long annealing time. For example, Gordon and Vandermeer's work on $40 \%$ cold-rolled aluminum alloys showed that the JMAX slopes fall below the straight line at long annealing time [50]. In contrast, our simulation suggests that the JMAX slope shows a strong positive deviation at fission density above $4.5 \times 10^{21} \mathrm{~cm}^{-3}$. Such a discrepancy might be ascribed to two reasons: the first one is the non-random distribution of nuclei and the second one is the formation of secondary phases, i.e., gas bubble phases in this case. The inclusion of secondary phases can cause the overestimation of the volume of recrystallized grains. To exclude the effect of gas bubbles, we recalculated the total volume fraction of recrystallization as a function of fission density without bubble phases, as shown in Fig. 4(b). As expected, the recalculated logarithmic plot shows a negative deviation at high fission density, indicating a decreased Avrami exponent $n$ at high fission density, which is consistent with the experiments. The linear part gives a value of 2.81 for the Avrami exponent $n$. It should be noted that the Avrami model is the simplest model for description of recrystallization kinetics and may not capture the non-Avrami behavior in irradiation-induced recrystallization process. A more complex model [51] may be needed to better describe the non-Avrami behavior during recrystallization, which is beyond the scope of this work. 
To study the effect of fission rate on the recrystallization kinetics, we performed additional simulations of recrystallization in U-7Mo alloy with two different fission rates, $5.0 \times 10^{14}$ and $7.0 \times 10^{14} \mathrm{~cm}^{-3} \mathrm{~s}^{-1}$, which are higher than the average fission rate typically used for in-pile radiation of U-Mo fuels. The initial grain structures for these two cases are the same as the one used for the simulation with fission rate of $3.0 \times 10^{14} \mathrm{~cm}^{-3} \mathrm{~s}^{-1}$ in the preceding section. As shown in Fig. 5, the recrystallized grain volume fractions are almost the same at low fission densities and have minor differences at high fission densities. A large fission rate leads to slightly higher recrystallization volume faction at the same fission density. For example, the recrystallized fuel volume fraction is 0.66 , 0.70 , and 0.73 at $4.5 \times 10^{21} \mathrm{~cm}^{-3}$ at the fission rate of 3.0, 5.0, and $7.0 \times 10^{14} \mathrm{~cm}^{-3} \mathrm{~s}^{-1}$, respectively. The full recrystallization is achieved at $5.5,5.4$, and $5.3 \times 10^{21} \mathrm{~cm}^{-3}$ for the fission rate of 3.0, 5.0, and $7.0 \times 10^{14} \mathrm{~cm}^{-3} \mathrm{~s}^{-1}$, respectively. These results indicate that 
fission rate has a negligible impact on the recrystallization kinetics in U-Mo alloy fuels, a result consistent with the conclusion from the rate theory model by Rest [3] and experimental analysis by Kim et al. [4].

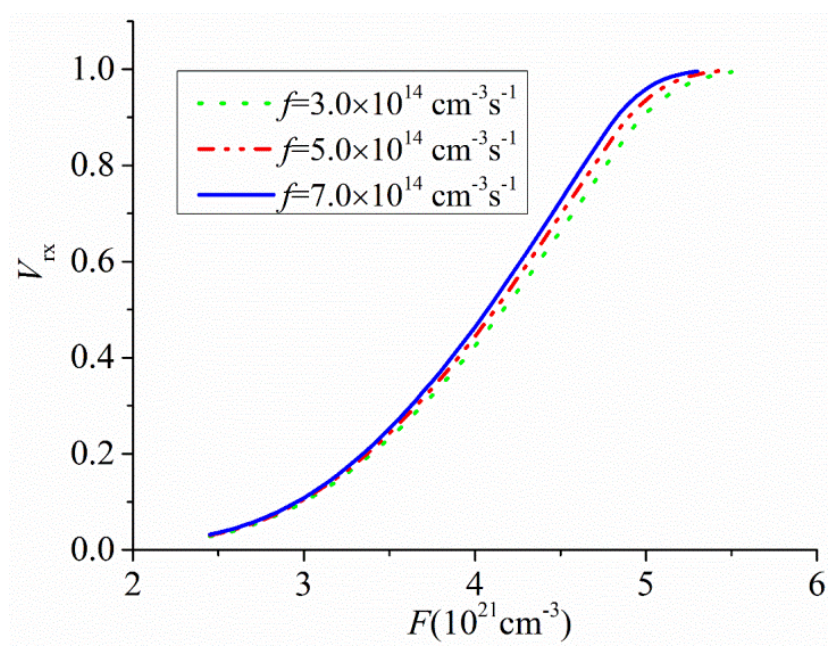

Fig. 5. Effect of fission rate on the recrystallization volume fraction of U-7Mo alloy.

\subsection{Effect of grain size on recrystallization}

The initial grain size can play an important role in the fission-induced recrystallization and swelling of nuclear materials. Unfortunately, few experimental studies have been done on the effect of grain size for U-Mo alloy fuels. The initial grain size is expected to affect the rate of recrystallization in several ways. For example, grain boundaries are favored nucleation sites, and therefore the number of available nucleation sites is greater for a fine-grained material. Generally, a fine-grained material recrystallizes more rapidly than does a coarse-grained material [52]. 
In this work, the grain boundary is the main factor that affects the recrystallization kinetics, since the stored energy and deformation inhomogeneities are considered the same for different grain size structures. The small grain area can reduce the propagation rate of the recrystallization front, and the short grain boundary length can decrease the possibility of the formation of recrystallized nuclei. To study the grain size effect, we constructed two additional grain structures with relatively larger original grain size, $5 \mu \mathrm{m}$ and $9.6 \mu \mathrm{m}$. The fission rate was set as $3.0 \times 10^{14} \mathrm{~cm}^{-3} \mathrm{~s}^{-1}$ the same as the case with $3 \mu \mathrm{m}$ grain size. To evaluate the effect of grain boundary on the recrystallization, we estimated the grain boundary length in the initial microstructure, which is defined as the summation of all voxels along the grain boundary with the grain parameter $\eta_{i}$ from 0.1 to 0.9 . The measured grain boundary lengths are $1.27 \times 10^{3}, 7.3 \times 10^{2}$, and $3.68 \times 10^{2} \mu \mathrm{m}$ for the original grain sizes of $3 \mu \mathrm{m}, 5 \mu \mathrm{m}$, and $9.6 \mu \mathrm{m}$, respectively. As expected, the grain boundary length decreases as the initial grain size increases. The simulated grain microstructures of these three cases irradiated up to a fission density of $4.1 \times 10^{21} \mathrm{~cm}^{-3}$ are shown in Fig. 6. One can see that more recrystallized nucleated grains form in the case with smaller initial grain size because of its larger grain boundary length. The smaller grain size can also reduce the time for the propagation of recrystallized front. The average sizes of the recrystallized grains are $0.33,0.28$, and $0.26 \mu \mathrm{m}$ for the original grain size of $3 \mu \mathrm{m}, 5 \mu \mathrm{m}$, and $9.6 \mu \mathrm{m}$, respectively. These results indicate that the recrystallized grain size is relatively large in the fuel with small initial grain size. The reason is that the large grain boundary length results in more recrystallized grains contacted by the initial grains. It is believed that the front of recrystallized grain has a larger driving force that includes the grain boundary energy and stored energy between 
the subgrains and initial grains, whereas the recrystallized grains have a smaller driving force that is attributed to the grain boundary energy. Thus, the recrystallized grains grow relatively faster in the small initial grain size structure.

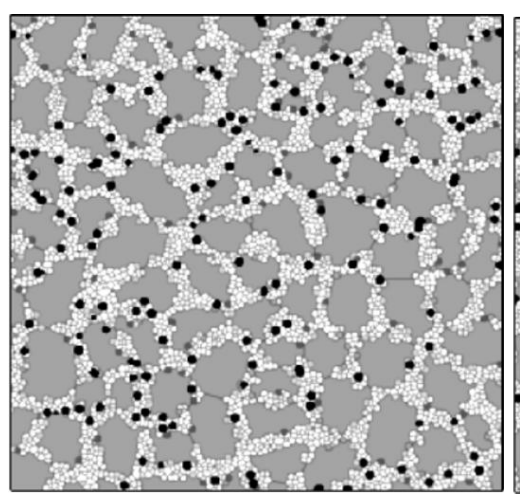

(a)

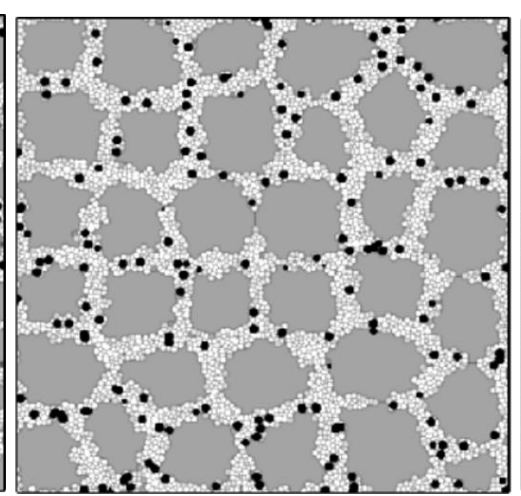

(b)

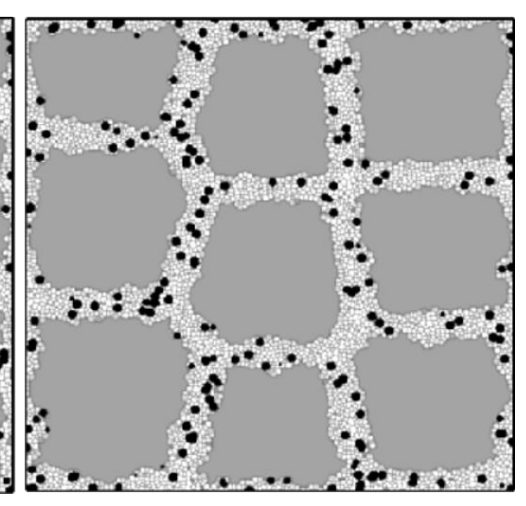

(c)

Fig. 6. Simulated grain microstructures of U-7Mo alloy with different initial grain sizes (a) $3.0 \mu \mathrm{m}$; (b) $5.0 \mu \mathrm{m}$; (c) $9.6 \mu \mathrm{m}$ at fission density of $4.1 \times 10^{21} \mathrm{~cm}^{-3}$.

To study the effect of the initial grain size on the rate of recrystallization, we compared the volume fraction of the recrystallized grains as a function of fission density for three different initial grain microstructures. As shown in Fig. 7(a), the case with the smallest initial grain has the largest volume fraction of recrystallized grains, and this effect becomes more prominent at high fission density. Accordingly, the full recrystallization in the microstructure with the largest grain size is achieved at the highest fission density of $6.4 \times 10^{21} \mathrm{~cm}^{-3}$, compared with the fission density of $5.5 \times 10^{21} \mathrm{~cm}^{-3}$ and $5.9 \times 10^{21} \mathrm{~cm}^{-3}$ needed for the other two cases with smaller initial grain sizes. To estimate the effect of grain size on recrystallization kinetics, we compared the simulation results 
with the JMAK model using a logarithmic plot as shown in Fig. 7(b). The Avrami exponent $n$ is determined to be $2.81,2.75$, and 2.55 for the microstructures with the initial grain size of 3.0, 5.0, and $9.6 \mu \mathrm{m}$, respectively, indicating that the rate of recrystallization is reduced because of the increase in the initial grain size. We point out that the recrystallization kinetics in the microstructure with the largest initial grain size of $9.6 \mu \mathrm{m}$ shows a strong negative deviation from the ideal JMAK model at high fission density, consistent with the previous study of the recrystallization kinetics of cold-rolled copper with different initial grain sizes [52].
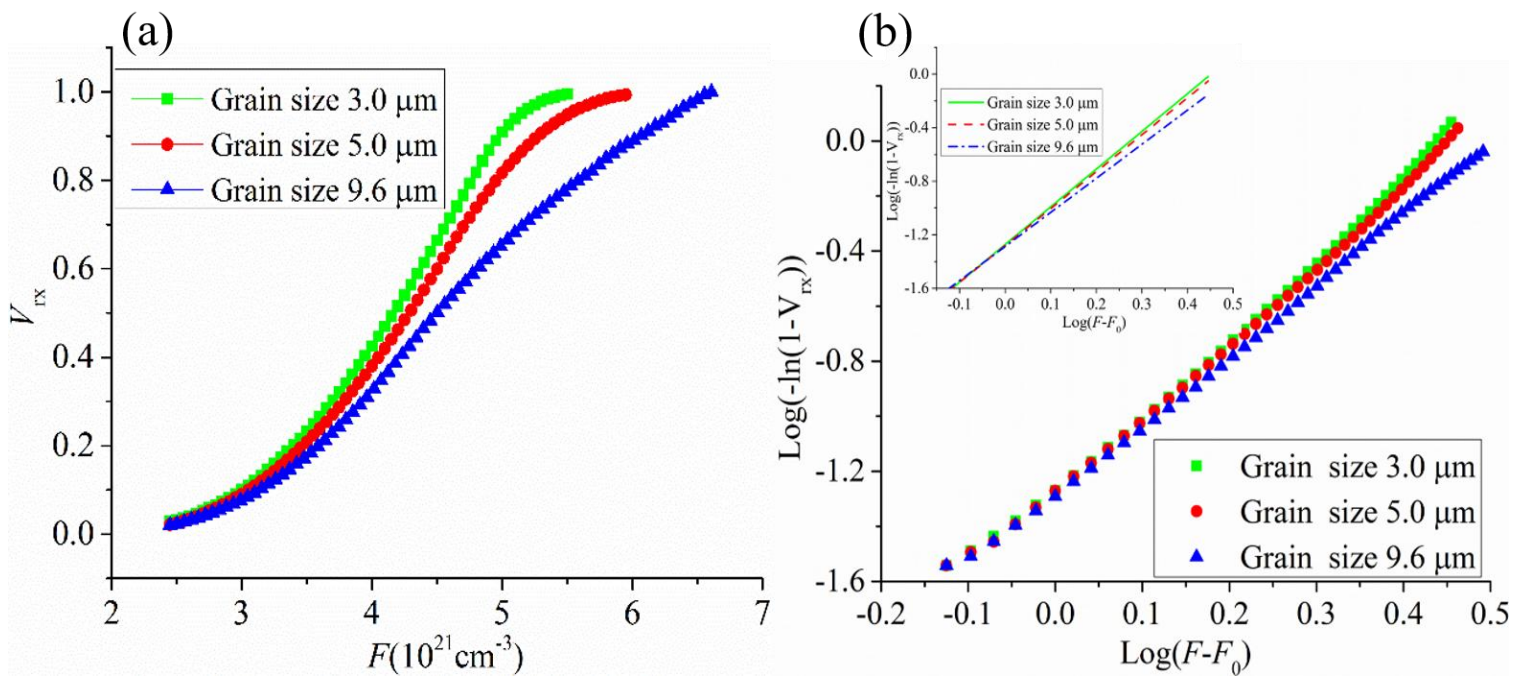

Fig. 7. (a) Recrystallized volume fractions of U-7Mo alloy as a function of fission density for three different initial grain sizes; (b) JMAX plot of the recrystallization kinetics. The insert is the linear fit of the JMAX plot.

To analyze quantitatively the effect of grain size on the recrystallization, we plotted the recrystallized volume fractions as a function of initial grain size at different fission densities in Fig. 8. At low fission density, the recrystallization volume fraction 
decreases almost linearly as a function of the initial grain size. The linear relationship can be explained by the linear dependence of the recrystallization rate on the initial grain boundary length. At high fission density, however, the recrystallized volume fraction does not follow the linear relationship anymore. The reason might be that the initial grain boundaries are fully consumed at high fission density and therefore the new formed grain boundary length fully does not have a simple linear relationship with the initial grain size.

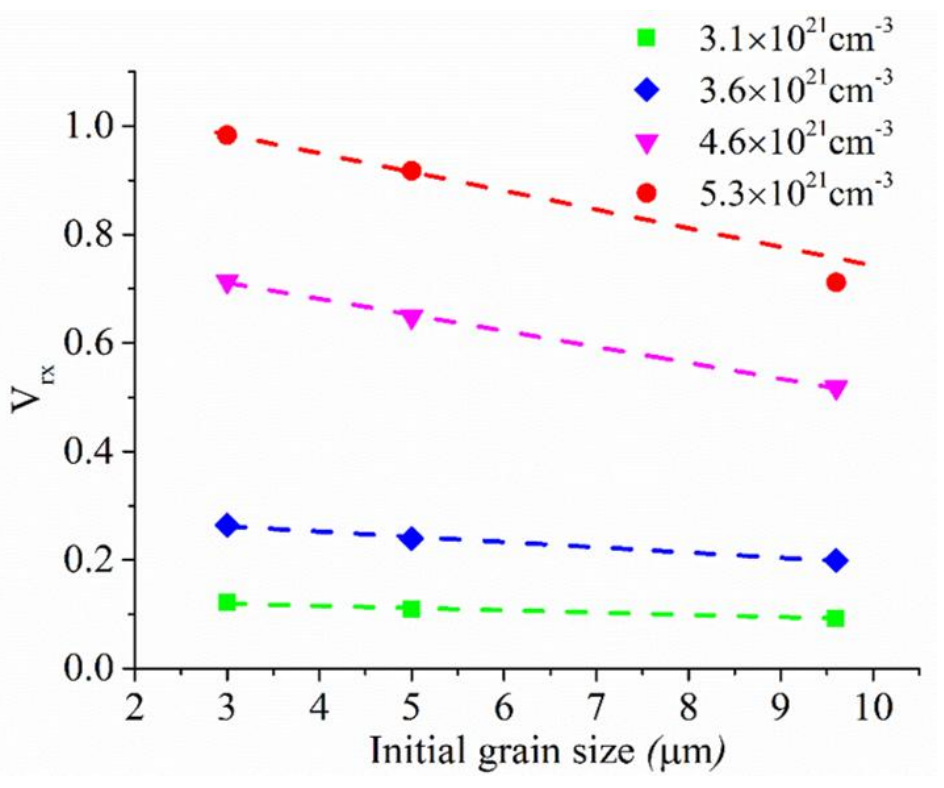

Fig. 8. Recrystallized volume fractions of U-7Mo alloy as a function of initial grain sizes at different fission densities.

\subsection{Effect of grain aspect ratio on recrystallization}

During the fabrication of monolithic U-Mo fuel plates, the fuel plates are hotrolled in order to achieve the desired plate thickness and better mechanical bonding between U-Mo fuel layer, $\mathrm{Zr}$ diffusion barrier layer, and Al cladding. After hot rolling, the grains in the fuel layer are found to be elongated along the rolling direction, resulting 
in different grain aspect ratios in the fuel [53]. Thus, the grain morphology and grain boundary in the monolithic U-Mo fuels differ significantly from that in the atomized UMo dispersion fuels and are expected to have a great impact on the recrystallization kinetics in U-Mo alloys. Moreover in atomized U-Mo fuel particles, columnar-shaped grains often form the outer layer of the atomized fuel particles as a result of fast cooling during the atomization process, while equal axial grains form inside the particles [54]. Therefore, it is of critical importance to understand the effect of grain morphology on the recrystallization in both monolithic and dispersion fuels of U-Mo.

Figure 9 shows the simulated recrystallized grain microstructures with three different aspect ratios of the initial grains, 1:1, 1:3, and 1:8. The average grain size is set the same, at $5.0 \mu \mathrm{m}$. After the initial elongated grain structures are generated, the grain growth kinetics of the three cases are controlled by the conventional Allen-Cahn kinetics. For the normal grain structure, the newly formed subgrains are nucleated on the grain boundaries equally in all directions. For an elongated grain structure, however, more subgrains are formed on the grain boundaries parallel to the elongated direction than on the grain boundaries perpendicular to that direction. At a fission density of $3.8 \times 10^{21} \mathrm{~cm}^{3}$, considerable grain boundaries along the elongated direction are not fully recrystallized, as shown in Figs. 9(b) and 9(c). This phenomenon is more prominent for the grain structure with a larger grain aspect ratio, as shown in Fig. 9(c). This can be attributed to the increased grain boundary length in the grain structure with increasing grain aspect ratio due to hot rolling, and therefore the increased nucleation density of recrystallization nuclei. Our simulations also indicate that the much shorter grain boundaries perpendicular to the elongated direction are fully recrystallized earlier than the longer 
grain boundaries parallel to the elongated direction, a result that is consistent with the fact that the triple points in grain structure are favored nucleation sites for recrystallization.

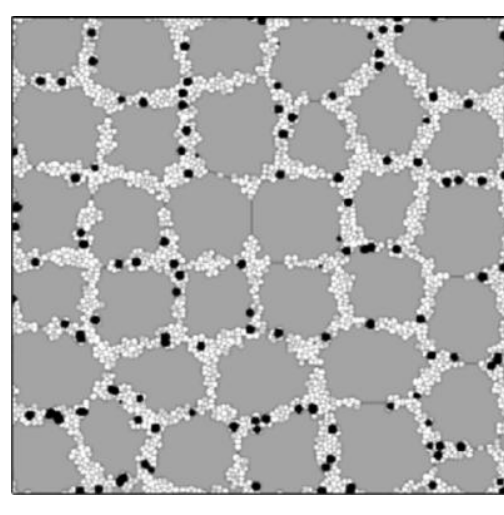

(a)

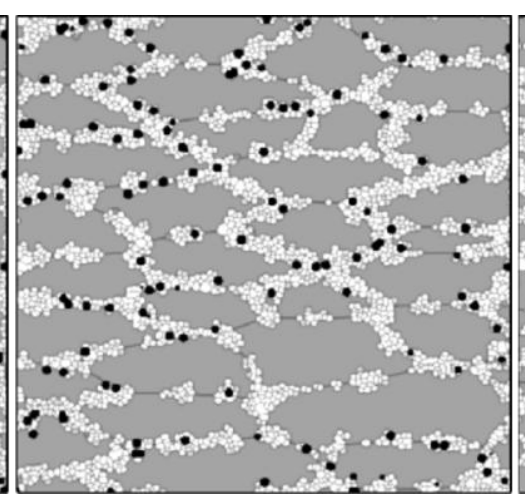

(b)

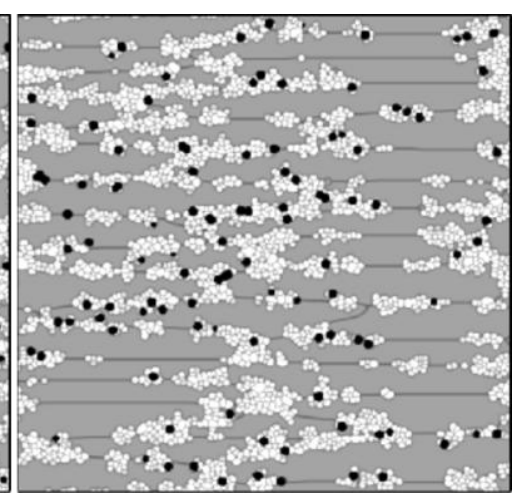

(c)

Fig. 9. Simulated grain microstructures of U-7Mo alloy with different initial grain aspect ratios (a) $1: 1$; (b) $1: 3$; (c) $1: 8$ at a fission density of $3.8 \times 10^{21} \mathrm{~cm}^{-3}$.

We also investigated the effect of grain aspect ratio on the recrystallization kinetics of U-7Mo alloys as a function of fission density. As shown in Fig. 10(a). The grain structure with the largest aspect ratio of the initial grains shows the highest rate of recrystallization. Full recrystallization is achieved at a fission density of 5.4, 5.7, and $6.0 \times 10^{21} \mathrm{~cm}^{-3}$ for the aspect ratio of $1: 8,1: 3$, and 1:1, respectively. Figure $10(\mathrm{~b})$ shows the JMAX plot of the recrystallization kinetic. From the linear fitting of the recrystallization data, the Avrami exponent $n$, is predicted to be $2.81,2.84$, and 2.93 for the initial grain aspect ratios of 1:1, 1:3, and 1:8 $\mu \mathrm{m}$, respectively. The increased value of the Avrami exponent due to the increased grain aspect ratio also is consistent with the conclusion that increased grain aspect ratio can lead to a higher recrystallization rate. 


\section{Conclusions}

A mesoscale model was developed by integrating the rate theory and phase-field model and was used to study the fission-induced recrystallization in U-7Mo alloys. The phase-field simulations can reproduce well the microstructure evolution during the grain subdivision process. The predicted fission density dependence of the recrystallized volume fraction agrees well with the experimental data. The Avrami exponent $n$ was determined to be 2.81 from the JMAK plot, which is close to the experimental value. Consistent with previous theoretical and experimental studies, the fission rate appears to have only a minor effect on the recrystallization in U-Mo alloy fuels. The grain morphology in the initial microstructure is found to have a significant impact on the recrystallization kinetics, especially the initial grain size. Increased initial grain size in the microstructure can notably reduce the rate of recrystallization and therefore delay the full 
recrystallization to a higher fission density. The effect of grain size on the recrystallization kinetics can be correlated with the grain boundary length. Moreover, our results show that a high grain aspect ratio of initial microstructure is not favored because of the increased grain boundary length compared with the grains with 1:1 ratio. It should be pointed out that the effect of the grain aspect ratio on the recrystallization kinetics of the U-7Mo alloy is considerably less prominent than that of grain size. Therefore, it is desired to increase the grain size and reduce the grain aspect ratio in the U-Mo dispersion and monolithic fuels in order to suppress the fuel recrystallization and therefore fuel swelling. We believe the present study of fission-induced recrystallization will be useful for improving the fuel performance of U-Mo alloys.

We point out some of the limitations of the models used in this work: (1) the nucleation processes of subgrains and fission gas bubbles are not considered in the current model; (2) the recrystallization in different grains is treated homogeneously; (3) the recrystallization is assumed to be static (i.e., the dislocation density does not evolve with time); and (4) other defects such as vacancies and self-interstitial atoms are not considered. We plan to address these issues in our further work.

\section{Acknowledgments}

This work was supported by the U.S. Department of Energy, National Nuclear Security Administration (NNSA), Office of Material Management and Minimization (NA-23) Reactor Conversion Program. Use of the Center for Nanoscale Materials, an Office of Science user facility, was supported by the U.S. Department of Energy, Office of Science, 
Office of Basic Energy Sciences, under Contract No. DE-AC02-06CH11357. We also acknowledge use of Fusion, a high-performance computing cluster operated by the Laboratory Computing Resource Center at Argonne National Laboratory. In addition, this manuscript benefited from insightful conversations with Jeff Rest.

\section{Reference}

[1] Y.S.K. G.L. Hofman, M.R. Finlay, J.L. Snelgrove, S.L. Hayes, M.K. Meyer, C.R. Clark, F. Huet, , in: the 25th International Meeting on Reduced Enrichment for Research and Test Reactors (RERTR), Chicago, Illinois, 2003.

[2] J. Rest, G.L. Hofman, Y.S. Kim, Journal of Nuclear Materials, 385 (2009) 563-571.

[3] J. Rest, Journal of Nuclear Materials, 346 (2005) 226-232.

[4] Y.S. Kim, G.L. Hofman, J.S. Cheon, Journal of Nuclear Materials, 436 (2013) 14-22.

[5] Y.S. Kim, J.M. Park, K.H. Lee, B.O. Yoo, H.J. Ryu, B. Ye, Journal of Nuclear Materials, 454 (2014) 238-246.

[6] Z.-G. Mei, L. Liang, Y.S. Kim, W. Tom, E.O. Hare, A.M. Yacout, G. Hofman, M. Anitescu, to be published.

[7] J. Rest, G.L. Hofman, Journal of Nuclear Materials, 210 (1994) 187-202.

[8] L.E. Thomas, C.E. Beyer, L.A. Charlot, Journal of Nuclear Materials, 188 (1992) 80-89.

[9] H. Matzke, Journal of Nuclear Materials, 189 (1992) 141-148.

[10] M. Kinoshita, Journal of Nuclear Materials, 248 (1997) 185-190.

[11] C.B. Lee, Y.H. Jung, Journal of Nuclear Materials, 279 (2000) 207-215.

[12] F.J. Humphreys, Scripta Metallurgica Et Materialia, 27 (1992) 1557-1562.

[13] E.A. Holm, M.A. Miodownik, A.D. Rollett, Acta Materialia, 51 (2003) 2701-2716.

[14] L. Sieradzki, L. Madej, Computational Materials Science, 67 (2013) 156-173.

[15] G. Kugler, R. Turk, Computational Materials Science, 37 (2006) 284-291.

[16] F. Han, B. Tang, H. Kou, L. Cheng, J. Li, Y. Feng, Journal of Materials Science, 49 (2014) 32533267.

[17] J. Rest, Journal of Nuclear Materials, 326 (2004) 175-184.

[18] J. Rest, Journal of Nuclear Materials, 349 (2006) 150-159.

[19] T. Takaki, A. Yamanaka, Y. Higa, Y. Tomita, Journal of Computer-Aided Materials Design, 14 (2007) 75-84.

[20] T. Takaki, Y. Tomita, International Journal of Mechanical Sciences, 52 (2010) 320-328.

[21] Y. Suwa, Y. Saito, H. Onodera, Materials Science and Engineering a-Structural Materials Properties Microstructure and Processing, 457 (2007) 132-138.

[22] Y. Suwa, Y. Saito, H. Onodera, Computational Materials Science, 44 (2008) 286-295.

[23] M. Muramatsu, Y. Aoyagi, Y. Tadano, K. Shizawa, Computational Materials Science, 87 (2014) 112-122.

[24] O. Guevenc, M. Bambach, G. Hirt, Steel Research International, 85 (2014) 999-1009.

[25] B. Zhu, M. Militzer, Modelling and Simulation in Materials Science and Engineering, 20 (2012).

[26] A. Vondrous, P. Bienger, S. Schreijaeg, M. Selzer, D. Schneider, B. Nestler, D. Helm, R. 
Moenig, Computational Mechanics, 55 (2015) 439-452.

[27] S.P. Gentry, K. Thornton, lop, Simulating recrystallization in titanium using the phase field method, in: 36th Riso International Symposium on Materials Science, 2015.

[28] L.Q. Chen, Annual Review of Materials Research, 32 (2002) 113-140.

[29] M. Wang, B.Y. Zong, G. Wang, Journal of Materials Science \& Technology, 24 (2008) 829834.

[30] S. Sreekala, M. Haataja, Physical Review B, 76 (2007).

[31] T. Takaki, T. Hirouchi, Y. Hisakuni, A. Yamanaka, Y. Tomita, Materials Transactions, 49 (2008) 2559-2565.

[32] T. Takaki, Y. Hisakuni, T. Hirouchi, A. Yamanaka, Y. Tomita, Computational Materials Science, 45 (2009) 881-888.

[33] K. Nogita, K. Une, Nuclear Instruments \& Methods in Physics Research Section B-Beam Interactions with Materials and Atoms, 91 (1994) 301-306.

[34] F.J. Humphreys, Acta Metallurgica, 25 (1977) 1323-1344.

[35] F.J. Humphreys, M. Hatherly, Recrystallization and Related Annealing Phenomena, First Edition ed., Elsevier Science Ltd, U. K, 1995.

[36] I. Steinbach, Annual Review of Materials Research, Vol 43, 43 (2013) 89-107.

[37] J. Rest, G.L. Hofman, Journal of Nuclear Materials, 277 (2000) 231-238.

[38] L. Liang, Z.-G. Mei, Y.S. Kim, T. Wiencek, G. Hofman, M. Anitescu, A.M. Yacout, in, 2015.

[39] J.F. Ziegler, M.D. Ziegler, J.P. Biersack, Nuclear Instruments and Methods in Physics Research Section B: Beam Interactions with Materials and Atoms, 268 (2010) 1818-1823.

[40] R.E. Stoller, M.B. Toloczko, G.S. Was, A.G. Certain, S. Dwaraknath, F.A. Garner, Nuclear Instruments and Methods in Physics Research Section B: Beam Interactions with Materials and Atoms, 310 (2013) 75-80.

[41] N. Moelans, F. Wendler, B. Nestler, Computational Materials Science, 46 (2009) 479-490.

[42] C.E. Krill, L.Q. Chen, Acta Materialia, 50 (2002) 3057-3073.

[43] R.D. Kamachali, I. Steinbach, Acta Materialia, 60 (2012) 2719-2728.

[44] S.G. Kim, D.I. Kim, W.T. Kim, Y.B. Park, Physical Review E, 74 (2006).

[45] N. Moelans, B. Blanpain, P. Wollants, Physical Review B, 78 (2008).

[46] L.Q. Chen, J. Shen, Computer Physics Communications, 108 (1998) 147-158.

[47] Y. Miao, K. Mo, B. Ye, L. Jamison, Z.-G. Mei, J. Gan, B. Miller, J. Madden, J.-S. Park, J. Almer, S. Bhattacharya, Y.S. Kim, G.L. Hofman, A.M. Yacout, Scripta Materialia, 114 (2016) 146-150.

[48] B.D. Miller, J. Gan, D.D. Keiser, Jr., A.B. Robinson, J.F. Jue, J.W. Madden, P.G. Medvedev, Journal of Nuclear Materials, 458 (2015) 115-121.

[49] R. Ho Jin, K. Yeon Soo, G.L. Hofman, J. Rest, P. Jong Man, K. Chang Kyu, Materials Science Forum, 558-559 (2007) 319-322.

[50] P. Gordon, R.A. Vandermeer, Trans. TMS-AIME, 224 (1962) 917.

[51] P. Saidi, S. Shahandeh, J.J. Hoyt, Metallurgical and Materials Transactions a-Physical Metallurgy and Materials Science, 46A (2015) 2975-2985.

[52] W.B. Hutchinson, S. Jonsson, L. Ryde, Scripta Metall., 23 (1989) 671.

[53] D.D. Keiser, J.F. Jue, B. Miller, W. Williams, F.J. Rice, A. Robinson, B. Rabin, M.K. Meyer, in: Research Reactor Fuel Management Conference, Bucharest, Romania, 2015.

[54] Y.S. Kim, G.L. Hofman, J. Rest, G.V. Shevlyakov, in, Argonne National Laboratory, 2008.

The submitted manuscript has been created by UChicago Argonne, LLC, Operator of Argonne National Laboratory ("Argonne"). Argonne, a U.S. Department of Energy 
Office of Science laboratory, is operated under Contract No. DE-AC02-06CH11357. The U.S. Government retains for itself, and others acting on its behalf, a paid-up nonexclusive, irrevocable worldwide license in said article to reproduce, prepare derivative works, distribute copies to the public, and perform publicly and display publicly, by or on behalf of the Government. The Department of Energy will provide public access to these results of federally sponsored research in accordance with the DOE Public Access Plan. http://energy.gov/downloads/doe-public-access-plan. 
Grain structures irradiated at a fission density of $4.1 \times 10^{21} \mathrm{~cm}^{3}$

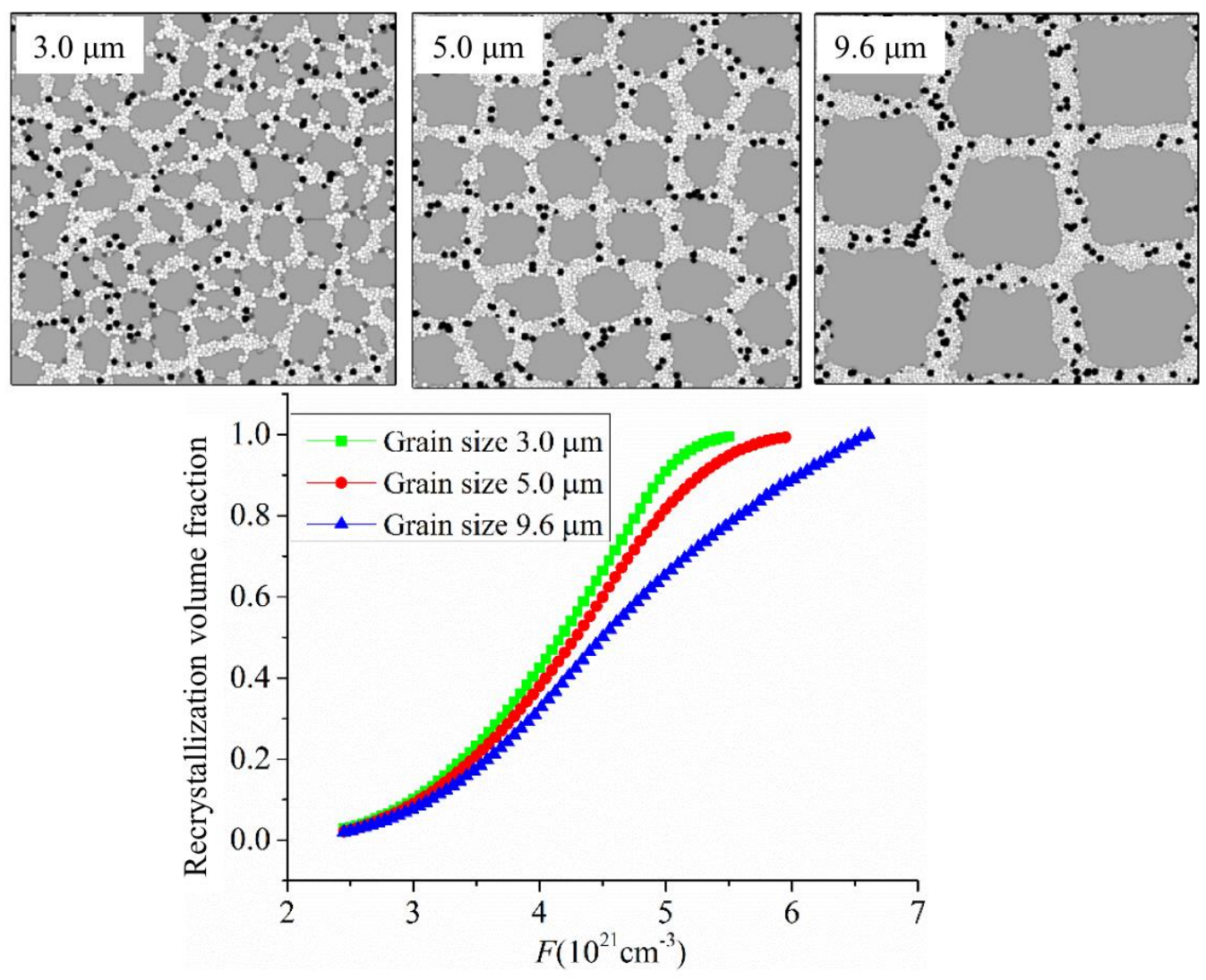

